\title{
Valproate and spinal muscular atrophy (Review)
}

\author{
GEMMA NATASHA, KEVIN G. BRANDOM, ELIZABETH C. YOUNG and PHILIP J. YOUNG \\ Clinical Neurobiology, Peninsula College of Medicine and Dentistry, St Luke's Campus, Exeter EX1 2LU, UK
}

Received December 14, 2007; Accepted January 11, 2008

\begin{abstract}
Childhood spinal muscular atrophy (SMA) is an autosomal recessive disorder caused by mutations in the survival motor neuron $(S M N)$ gene. The severity of the disease is dictated by the copy number of a second copy of the gene, known as $S M N 2$, with higher copy numbers associated with milder forms of SMA. This is because the level of SMN protein produced by patients dictates the severity of the disease. As all patients retain at least one copy of the SMN2 gene, therapeutic strategies are geared towards increasing full-length SMN protein expression from SMN2. One of the identified therapeutic compounds is valproic acid, or valproate (VPA), a histone deacetylase inhibitor (HDACI) that has been used since the 1970s as an anti-convulsant. Here, we discuss VPA's modes of action and potential side effects in the treatment of SMA.
\end{abstract}

\section{Contents}

1. Introduction

2. VPA and other HDACIs as potential therapeutic agents for childhood SMA

3. VPA alters the expression of various neurotransmitters

4. VPA affects $\gamma$-aminobutric acid levels

5. VPA affects amino acid neurotransmitters

6. VPA modulates monoamines

7. VPA inhibits the high frequency firing of neurons

8. VPA alters the cerebral glucose metabolism

9. VPA stimulates intracellular signalling pathways

10. Pharmacological profile of VPA and its implications for the treatment of SMA

\section{Introduction}

Childhood spinal muscular atrophy (SMA) is an autosomal recessive disorder characterised by the loss of the $\alpha$-motor neurons of the spinal cord (1). SMA is caused by mutations

Correspondence to: Dr Philip J. Young, Clinical Neurobiology, Peninsula College of Medicine and Dentistry, St Luke's Campus, Exeter EX1 2LU, UK

E-mail: philip.young@pms.ac.uk

Key words: valproate, spinal muscular atrophy of the survival motor neuron (SMN) gene (1). Humans are the only species to have two copies of the SMN gene: SMN1 and SMN2 (Fig. 1). The two forms of the gene differ by only 11 nucleotides, one of which disrupts an exonic splicing enhancer in $S M N$ exon 7, resulting in an aberrant splicing event that excises exon 7 from $90 \%$ of SMN2-derived transcripts (2-4) (Fig. 1). As the shorter form of the protein lacking exon 7 (SMN $\Delta 7$ ) is unstable, effectively only $10 \%$ of the protein derived from the SMN2 gene is functional $(1,5)$ (Fig. 1).

Full-length SMN (FL-SMN) protein levels dictate SMA onset and severity $(6,7)$. Therefore, the expression of SMN1 ensures a normal phenotype $(6,7)$. Accordingly, individuals harbouring mutated SMN2 genes who retain an intact SMN1 gene display no clinical symptoms of SMA (1). This is because the amount of FL-SMN expressed by the SMN1 gene ensures the viability of all cell types, including motor neurons $(6,7)$. However, in SMA patients the SMN1 gene is either deleted or mutated, resulting in a significant reduction in FL-SMN levels $(6,7)$.

Although the SMN2 gene cannot completely compensate for the loss of SMN1 in SMA, SMN2 copy number has been shown to have an inverse relationship with disease severity $(8,9)$. These findings suggest that the differing phenotypes of SMA, type I, II and III, are mediated by SMN protein levels, with type III patients having a higher SMN2 copy number than type I patients, thus expressing more FL-SMN protein (10). As all patients retain at least one copy of $S M N 2$, and as increased SMN protein levels reduce disease severity, compounds that increase total transcription levels from the SMN2 gene are considered to be legitimate therapeutic agents.

\section{VPA and other HDACIs as potential therapeutic agents for childhood SMA}

Initial screening of therapeutic agents identified several histone deacetylase inhibitors (HDACIs) as compounds capable of increasing FL-SMN expression at both the RNA and protein levels (11-15). Histone deacetylases remove acetyl groups from the histone tails, enabling the histone to wrap tightly around genomic DNA. This folding silences the gene by blocking the promoter regions and preventing the pre-initiation complex from forming (14). HDACIs cause a general increase in transcription by maintaining histones in an acetylated form, thus preventing chromatin folding and gene silencing (14).

One of the identified HDACIs is valporic acid, also known as valproate (VPA). VPA is a short-chained fatty acid originally synthesised as an analogue of valeric acid found in 


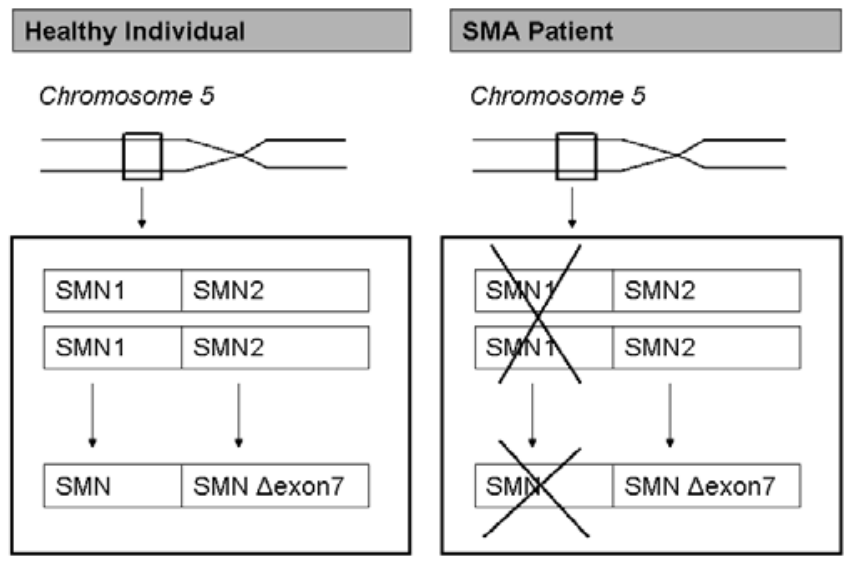

Figure 1. Schematic diagram showing the SMN gene locus in healthy individuals and SMA patients. Two nearly identical copies of the SMN gene are located on the long arm of chromosome 5 (5q13). Functional SMN protein is predominantly produced by SMN1, whereas the major product of SMN2 is a truncated and non-functional protein (SMN $\Delta$ exon7). Mutations that cause the disease inactivate SMN1, leaving SMN2 as the only source of functional protein.

the perennial Valeriana officinalis (16-18). An FDA-approved drug, it was inadvertently found to successfully prevent seizures, and has since been used to treat both generalised and partial seizures (16-18). The fact that VPA has been successful in treating such a wide range of seizures and, more recently, in treating pathologies such as migraine and bipolar disorder, suggests that it has a number of mechanisms of action (19). The most recently discovered mechanism of action, the inhibition of histone deacetylase, has raised the possibility that VPA could be used to treat other neurological pathologies, such as SMA (12).

Fibroblast cultures derived from patients with SMA and treated with therapeutic doses of VPA $(0.5-1000 \mu \mathrm{m})$ showed increased FL-SMN at both the RNA and protein levels (12). A greater increase in FL-SMN expression was observed in cells from patients with three rather than two copies of the SMN2 gene, suggesting that type III SMA patients, who have more copies of $S M N 2$, will exhibit a better response to treatment with VPA (12). The increase in FL-SMN protein was proposed to be due to correction of the SMN2 splicing pattern and an increase in SMN2 gene transcription (12). It has been proposed that the increase in SMN gene transcription is due to the activation of SMN promoters via AP1 and SP1 transcription factors (12).

It is now necessary to establish whether increased SMN protein levels are enough to prevent anterior horn motor neuron degeneration, or whether other factors contribute to the pathology of SMA. Studies with SMA mice suggest that this is the case, with increased SMN levels alone reducing disease severity (9). However, Gemins have been shown to modulate each other's expression, with RNAi knockdown of each individual Gemin changing the expression pattern of various SMN-binding partners (20). This suggests that Gemin proteins may auto-regulate their expression patterns (20). It is therefore possible that, by increasing SMN levels, VPA may also increase expression of all of Gemins, either by directly driving their expression through their own individual promoters, or by means of a secondary mechanism involving
SMN. Further studies are required to determine the exact effect of VPA on the expression profile of each Gemin.

\section{VPA alters the expression of various neurotransmitters}

As well as prolonging survival in SMA mice by increasing SMN protein levels, VPA and other HDACIs may confer neuronal protection by means of alternative mechanisms. In this review, we discuss these alternative mechanisms and their potential benefit to SMA.

\section{VPA affects $\gamma$-aminobutyric acid levels}

One altenative mechanism is the increase of $\gamma$-aminobutyric acid (GABA) at the nerve terminals (21-27). It has been proposed that VPA accomplishes this through the inhibition of the enzyme succinate semialdehyde dehydrogenase (SSA-DH) (21-27) (Fig. 2). This mechanism functions by creating a greater overall level of GABAergic inhibition in the cerebral cortex, which prevents the conduction of the abnormal excitatory glutamatergic nerve impulses observed in seizures (21-27). However, high and toxic doses of VPA inhibit glutamate decarboxylase (GAD) (21-27) (Fig. 2). GAD is the enzyme that converts glutamate to GABA; therefore its inhibition could decrease levels of GABA, increase levels of glutamate and cause seizures. This mechanism has implications for the use of VPA in SMA, because VPA is not equally distributed in the body when administered. For example, the plasma/brain ratio is $0.15-0.2$ following oral administration (24), demonstrating that most VPA is retained in the blood system and does not cross the blood brain barrier (22). This is important as it may mean that higher doses are needed to ensure that therapeutic levels of VPA are actually delivered to the motor neurons in the spinal cord.

\section{VPA affects amino acid neurotransmitters}

VPA also causes the inhibition of amino acid excitatory neurotransmission. This mechanism prevents any spikes in the excitatory nerve impulses that might cause seizures by reducing the levels of available excitatory neurotransmitter (28-32). The theory that VPA reduces the levels of excitatory amino acid aspartate (28-32) is supported by evidence that aspartate release is inhibited by VPA in a time- and dose-dependent manner (22). The reduction of glutamatergic excitatory transmission through the antagonism of the NMDA receptors has been proposed as the mechanism utilised by VPA in treating manic episodes (28-32). This may prevent the death of the injured motor neurons in SMA by preventing the excitotoxic effects of glutamate, which immature motor neurons are known to be more susceptible to. This mechanism of action may have other implications for the treatment of SMA with VPA in that, used chronically, it may cause side effects such as general psychomotor slowing if not delivered locally.

\section{VPA modulates monoamines}

VPA is also known to modulate monoamines (33-37). Chronic treatment with VPA has been shown to increase norepinephrine, dopamine and serotonin in both the brainstem and 


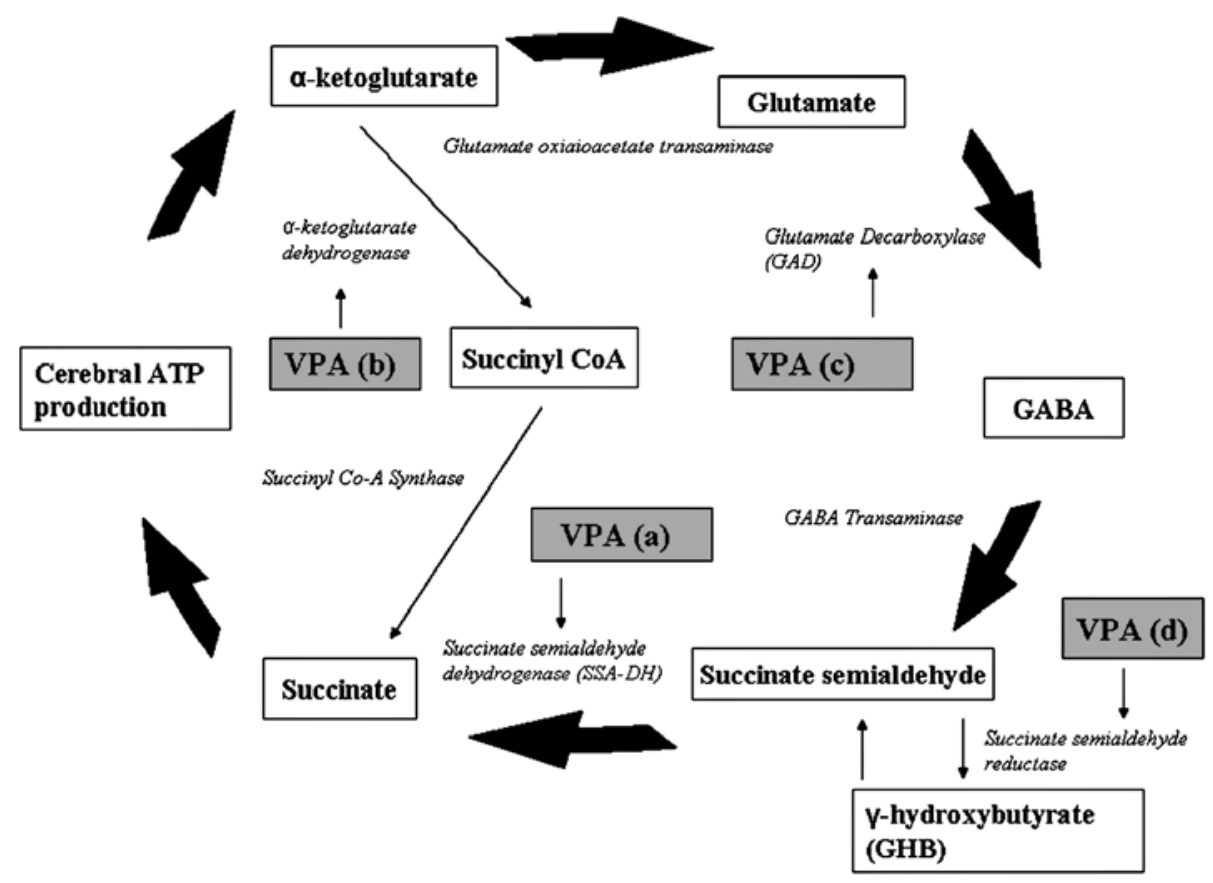

Figure 2. Flow diagram showing the mechanisms of action of VPA. (a) SSA-DH inhibition leads to high concentrations of its substrate succinate semialdehyde (SSA). This then leads to inhibition of the enzyme GABA transaminase (GABA-T) whose product is SSA. GABA-T breaks down GABA, and therefore its inhibition leads to higher levels of GABA. (b) High and toxic doses of VPA inhibit glutamate decarboxylase (GAD) leading to a decrease in levels of GABA and an increase in levels of glutamate. (c) VPA inhibits $\alpha$-ketoglutarate dehydrogenase, which leads to a decrease in the synthesis of cerebral ATP. (d) VPA inhibits the enzyme that produces $\gamma$-hydroxybutyrate (GHB), succinate semialdehyde reductase, and inhibits the calcium-dependent release of GHB.

the hippocampus (33-37). It has been proposed that increases in serotonin caused by VPA may affect glutamatergic excitatory neurotransmission through presynaptic regulation (33-37). This may be the mechanism by which VPA alleviates manic episodes, and is of import to SMA as altered levels of dopamine may induce the tremors associated with Parkinson's disease.

\section{VPA inhibits the high frequency firing of neurons}

At high concentrations, VPA inhibits the high frequency firing of neurons by blocking various voltage-gated ion channels (24). This function is believed to be the main mechanism by which VPA functions as an anti-convulsant (22). There are several ways in which VPA is believed to do this. At high concentrations, VPA first inhibits the opening of voltage-gated sodium channels (38) preventing efficient sodium influx and, therefore, reducing the generation of action potential and of firing frequencies. Second, also at high concentrations, it inhibits calcium influx in pre-synaptic neurons (39), thus inhibiting the release of neurotransmitters into the synaptic cleft and preventing synaptic transmission. The levels of VPA needed to trigger these side effects are relatively high; $1 \mathrm{mM}$ is needed to trigger the reduction in neuronal firing rates (24). Although increases in SMN protein have been detected in cultured cell lines treated with lower concentrations of VPA $(0.5-500 \mu \mathrm{M})$ (12), increases have only been observed in normal and SMA model animals treated with high VPA doses $(2 \mathrm{mM})(12,40)$. Treating SMA patients with high levels of VPA could therefore trigger synaptic transmission problems. However, these issues are not, for the most part, life threatening, and are consequently considered to be acceptable side effects.

\section{VPA alters the cerebral glucose metabolism}

Reports have demonstrated that the rate of cerebral glucose metabolism is clearly altered in people treated with VPA. This is believed to occur through the inhibition of $\alpha$-ketoglutarate dehydrogenase, which leads to a decrease in the synthesis of cerebral ATP (41-44). The inhibition of ATP production may be a contributing factor to the reduction of sodium channel conductance and, therefore, of neuronal excitability (41-44). This mechanism explains the effectiveness of VPA in treating epilepsy and mania, in which high levels of cerebral energy metabolism are observed. Decreases in glucose metabolism may also explain the cognitive impairment seen with chronic VPA use. Again, this has implications on the mode of delivery of VPA and the length of treatment for SMA.

\section{VPA stimulates intracellular signalling pathways}

Recently, it was shown that VPA triggers neurite outgrowth in treated PC12 cells at relatively low concentrations (500-1000 $\mu \mathrm{m})$. At these concentrations, SMN protein levels were not altered, suggesting that neurite outgrowth is triggered in an SMN-independent manner. It is proposed that VPA directly or indirectly stimulates the mitogen-activated protein kinase/extracellular signal-regulated kinase (MAPK/ERK) pathway $(45,46)$. This is a signal transduction pathway that links the binding of growth factors at cell-surface receptors to intracellular responses such as proliferation, differentiation, survival and apoptosis $(45,46)$. Other studies have shown that VPA exhibits neuroprotective effects by stimulating other intracellular signal pathways, such as the anti-apoptotic pathway that up-regulates the survival protein Bcl-2 (47). 


\section{Pharmacological profile of VPA and its implications for the treatment of SMA}

The half-life of VPA varies between species. Any acute effects of VPA noted in mouse $\left(\mathrm{t}_{1 / 2}=0.8 \mathrm{~h}\right)$ or rat $\left(\mathrm{t}_{1 / 2}=2-5 \mathrm{~h}\right)$ models cannot easily predict its effect on humans $\left(\mathrm{t}_{1 / 2}=9-18 \mathrm{~h}\right)$ (24). Brain concentrations of VPA in humans and mice are comparable, but concentrations of VPA in humans and rats are not (22). Care will therefore have to be taken in extrapolating the results of animal models to clinical trials in humans with SMA. The bioavailability of VPA is almost complete (96-100\%), but the time periods needed for it to reach peak concentrations vary between formulations. This may not be important in terms of the chronic treatment of SMA with VPA, but it would gain relevance were VPA to be administered in discrete doses in utero. Were VPA to be given as a chronic treatment to SMA patients, the fact that the protein-bound fraction is less in patients with renal or hepatic disease, in the elderly, during pregnancy and in the presence of other protein-bound drugs means that the monitoring of serum concentrations would have to be undertaken.

Although VPA follows first order kinetics, interindividual differences in metabolism have led to poor correlations between doses and serum concentrations (22). This again indicates that regular blood samples from patients would have to be analysed. Another important consideration is the fact that children require considerably higher doses of VPA per kilogram than adults to obtain comparable serum concentrations (22). Since the population treated for SMA are largely children, toxicity might be a problem. Doses of up to $1500 \mathrm{mg}$ have been utilised safely in migraine sufferers (22). It should be noted that VPA could also cause intoxication by inhibiting the metabolism of a number of drugs. The dosage of any comedication would therefore have to be adjusted as well, and serum concentrations monitored.

Dose-related side effects have included ones of a gastrointestinal, hepatic, haematological, neurological, metabolic and endocrine nature. All of these would have to be monitored, and might prevent VPA from becoming a mainstream treatment for SMA. The idiosyncratic side effect of VPA most likely to prevent it from becoming a widely used in utero treatment for SMA is its teratogenicity. The prevalence of neural tube defects in the offspring of mothers treated with VPA is $1-2.5 \%$ (22). This fact is likely to prompt the development of derivative compounds with reduced toxicity, such as SDP41 and valrocemide, and analogues of VPA such as valnoctamide, valpromide and valnotic acid. Whether any such derivatives or analogues would have the same efficacy in treating SMA is the topic of current and future research.

\section{Acknowledgements}

The authors wish to thank Dr Robert Morse for his comments and reviews. P.J.Y. is sponsored by the Vandervell Foundation, Fight SMA and IBCS.

\section{References}

1. Lefebvre S, Burglen L, Reboullet S, et al: Identification and characterization of a spinal muscular atrophy-determining gene. Cell 80: 155-165, 1995.
2. Lorson CL and Androphy EJ: An exonic enhancer is required for inclusion of an essential exon in the SMA-determining gene SMN. Hum Mol Genet 9: 259-265, 2000.

3. Lorson CL, Hahnen E, Androphy EJ and Wirth B: A single nucleotide in the SMN gene regulates splicing and is responsible for spinal muscular atrophy. Proc Natl Acad Sci USA 96: 6307-6311, 1999.

4. Monani UR, Lorson CL, Parsons DW, et al: A single nucleotide difference that alters splicing patterns distinguishes the SMA gene SMN1 from the copy gene SMN2. Hum Mol Genet 8: 1177-1183, 1999.

5. Lorson CL, Strasswimmer J, Yao JM, et al: SMN oligomerization defect correlates with spinal muscular atrophy severity. Nat Genet 19: 63-66, 1998.

6. Coovert DD, Le TT, McAndrew PE, et al: The survival motor neuron protein in spinal muscular atrophy. Hum Mol Genet 6: 1205-1214, 1997.

7. Lefebvre S, Burlet P, Liu Q, et al: Correlation between severity and SMN protein level in spinal muscular atrophy. Nat Genet 16: 265-269, 1997.

8. Monani UR, Coovert DD and Burghes AH: Animal models of spinal muscular atrophy. Hum Mol Genet 9: 2451-2457, 2000.

9. Monani UR, Sendtner M, Coovert DD, et al: The human centromeric survival motor neuron gene (SMN2) rescues embryonic lethality in $\operatorname{Smn}(-/-)$ mice and results in a mouse with spinal muscular atrophy. Hum Mol Genet 9: 333-339, 2000 .

10. Wirth B, Brichta L, Schrank B, et al: Mildly affected patients with spinal muscular atrophy are partially protected by an increased SMN2 copy number. Hum Genet 119: 422-428, 2006.

11. Avila AM, Burnett BG, Taye AA, et al: Trichostatin A increases SMN expression and survival in a mouse model of spinal muscular atrophy. J Clin Invest 117: 659-671, 2007.

12. Brichta L, Hofmann Y, Hahnen E, et al: Valproic acid increases the SMN2 protein level: a well-known drug as a potential therapy for spinal muscular atrophy. Hum Mol Genet 12: 2481-2489, 2003.

13. Hahnen E, Eyüpoglu IY, Brichta L, et al: In vitro and ex vivo evaluation of second-generation histone deacetylase inhibitors for the treatment of spinal muscular atrophy. J Neurochem 98: 193-202, 2006.

14. Kernochan LE, Russo ML, Woodling NS, et al: The role of histone acetylation in SMN gene expression. Hum Mol Genet 14: 1171-1182, 2005.

15. Riessland M, Brichta L, Hahnen E and Wirth B: The benzamide M344, a novel histone deacetylase inhibitor, significantly increases SMN2 RNA/protein levels in spinal muscular atrophy cells. Hum Genet 120: 101-110, 2006.

16. Eadie MJ: Could valerian have been the first anticonvulsant? Epilepsia 45: 1338-1343, 2004.

17. Eadie MJ, Heazlewood V, McKauge L and Tyrer JH: Steady-state valproate pharmacokinetics during long term therapy. Clin Exp Neurol 19: 183-191, 1983.

18. Eadie MJ, Tyrer JH, Smith GA and McKauge L: Pharmacokinetics of drugs used for petit mal 'absence' epilepsy. Clin Exp Neurol 14: 172-183, 1977.

19. Phiel CJ, Zhang F, Huang EY, Guenther MG, Lazar MA and Klein PS: Histone deacetylase is a direct target of valproic acid, a potent anticonvulsant, mood stabilizer, and teratogen. J Biol Chem 276: 36734-36741, 2001.

20. Shpargel KB and Matera AG: Gemin proteins are required for efficient assembly of Sm-class ribonucleoproteins. Proc Natl Acad Sci USA 102: 17372-17377, 2005.

21. Bialer M, Johannessen SI, Kupferberg HJ, Levy RH, Perucca E and Tomson T: Progress report on new antiepileptic drugs: a summary of the Seventh Eilat Conference (EILAT VII). Epilepsy Res 61: 1-48, 2004.

22. Johannessen CU and Johannessen SI: Valproate: past, present, and future. CNS Drug Rev 9: 199-216, 2003.

23. Johannessen SI and Ben-Menachem E: Management of focalonset seizures: an update on drug treatment. Drugs 66: 1701-1725, 2006.

24. Johannessen CU: Mechanisms of action of valproate: a commentatory. Neurochem Int 37: 103-110, 2000.

25. Perucca E and Johannessen SI: The ideal pharmacokinetic properties of an antiepileptic drug: how close does levetiracetam come? Epileptic Disord 5 (Suppl 1): 17-26, 2003.

26. Johannessen SI and Tomson T: Pharmacokinetic variability of newer antiepileptic drugs: when is monitoring needed? Clin Pharmacokinet 45: 1061-1075, 2006. 
27. Bialer M, Johannessen SI, Kupferberg HJ, Levy RH, Loiseau P and Perucca E: Progress report on new antiepileptic drugs: a summary of the Sixth Eilat Conference (EILAT VI). Epilepsy Res 51: 31-71, 2002.

28. Hassel B, Iversen EG, Gjerstad L and Tauboll E: Up-regulation of hippocampal glutamate transport during chronic treatment with sodium valproate. J Neurochem 77: 1285-1292, 2001.

29. Löscher W: In vivo administration of valproate reduces the nerve terminal (synaptosomal) activity of GABA amino-transferase in discrete brain areas of rats. Neurosci Lett 160: 177-180, 1993.

30. Löscher W: Effects of the antiepileptic drug valproate on metabolism and function of inhibitory and excitatory amino acids in the brain. Neurochem Res 18: 485-502, 1993 .

31. Löscher W and Hönack D: Effects of the competitive NMDA receptor antagonist, CGP 37849, on anticonvulsant activity and adverse effects of valproate in amygdala-kindled rats. Eur J Pharmacol 234: 237-245, 1993.

32. Valin A, Voltz C, Naquet R and Lloyd KG: Effects of pharmacological manipulation on neurotransmitter and other amino acid levels in the CSF of the Senegalese baboon Papio papio. Brain Res 538: 15-23, 1991.

33. Ahmad AM, Douglas Boudinot F, Barr WH, Reed RC and Garnett WR: The use of Monte Carlo simulations to study the effect of poor compliance on the steady state concentrations of valproic acid following administration of enteric-coated and extended release divalproex sodium formulations. Biopharm Drug Dispos 26: 417-425, 2005.

34. Baf MH, Subhash MN, Lakshmana KM and Rao BS: Sodium valproate induced alterations in monoamine levels in different regions of the rat brain. Neurochem Int 24: 67-72, 1994.

35. Narita N, Kato M, Tazoe M, Miyazaki K, Narita M and Okado N: Increased monoamine concentration in the brain and blood of fetal thalidomide- and valproic acid-exposed rat: putative animal models for autism. Pediatr Res 52: 576-579, 2002.

36. Owens MJ and Nemeroff CB: Pharmacology of valproate. Psychopharmacol Bull 37 (Suppl 2): 17-24, 2003.

37. Vriend JP and Alexiuk NA: Effects of valproate on amino acid and monoamine concentrations in striatum of audiogenic seizureprone Balb/c mice. Mol Chem Neuropathol 27: 307-324, 1996.
38. Yamamoto R, Yanagita T, Kobayashi H, Yokoo H and Wada A: Up-regulation of sodium channel subunit mRNAs and their cell surface expression by antiepileptic valproic acid: activation of calcium channel and catecholamine secretion in adrenal chromaffin cells. J Neurochem 68: 1655-1662, 1997.

39. Slater GE and Johnston D: Sodium valproate increases potassium conductance in Aplysia neurons. Epilepsia 19: 379-384, 1978.

40. Tsai LK, Tsai MS, Lin TB, Hwu WL and Li H: Establishing a standardized therapeutic testing protocol for spinal muscular atrophy. Neurobiol Dis 24: 286-295, 2006.

41. Di Daniel E, Cheng L, Maycox PR and Mudge AW: The common inositol-reversible effect of mood stabilizers on neurons does not involve GSK3 inhibition, myo-inositol-1-phosphate synthase or the sodium-dependent myo-inositol transporters. Mol Cell Neurosci 32: 27-36, 2006.

42. Morland C, Boldingh KA, Iversen EG and Hassel B: Valproate is neuroprotective against malonate toxicity in rat striatum: an association with augmentation of high-affinity glutamate uptake. J Cereb Blood Flow Metab 24: 1226-1234, 2004.

43. Rekling JC: Neuroprotective effects of anticonvulsants in rat hippocampal slice cultures exposed to oxygen/glucose deprivation. Neurosci Lett 335: 167-170, 2003.

44. Janssen F, Rambeck B and Schnabel R: Acute valproate intoxication with fatal outcome in an infant. Neuropediatrics 16: 235-238, 1985.

45. Van Bergeijk J, Haastert K, Grothe C and Claus P: Valproic acid promotes neurite outgrowth in $\mathrm{PC} 12$ cells independent from regulation of the survival of motoneuron protein. Chem Biol Drug Des 67: 244-247, 2006.

46. Van Bergeijk J, Rydel-Könecke K, Grothe C and Claus P: The spinal muscular atrophy gene product regulates neurite outgrowth: importance of the C terminus. FASEB J 21: 1492-1502, 2007.

47. Sugai F, Yamamoto Y, Miyaguchi K, et al: Benefit of valproic acid in suppressing disease progression of ALS model mice. Eur J Neurosci 20: 3179-3183, 2004. 